\title{
TRUTH GAPS, TRUTH GLUTS, AND THE LIAR PARADOX
}

\section{Jeremiah Joven B. Joaquin Philosophy Department, De La Salle University, Philippines}

\begin{abstract}
The liar paradox results from a line of reasoning that starts with the liar sentence, 'This sentence is false' and ends with a contradictory conclusion, 'The liar sentence is both true and false'. There have been solutions to the paradox that preserve the standard conception of truth and the classical notion of logical validity. In this paper, I explore nonstandard solutions to it. In particular, I focus on two non-classical solutions to the liar paradox; viz., the gappy and the glutty solutions. According to the gappy solution, the liar sentence is neither true nor false, and the reasoning that leads to the paradoxical conclusion is unsound. On the other hand, according to the glutty solution, the paradoxical conclusion is correct, but any subsequent reasoning from it is invalid. I show some ways of motivating each of these solutions. Next, I show what each implies about the notions of truth and validity, and how each solves the paradox. Finally, I highlight some of the more recent problems that could be pitted against each of these solutions.
\end{abstract}

\section{INTRODUCTION}

The liar paradox has challenged the standard conception of truth; so much so that logicians and philosophers, past and present, have scratched their heads ascertaining the truth of the liar sentence, 'This sentence is false.' Following the standard view of truth and falsity, the liar sentence leads to a paradox. The reasoning behind this is easy enough to follow.

If the liar sentence is true, then what it says holds. Since it says of itself that it is false, it follows then that it is false. Thus, if the liar sentence is true, then it is false. But if the liar sentence is false, then what it says does not hold. Since it says of itself that it is false, it follows then that it is true. Thus, if the liar sentence is false, then it is true. This simple line of reasoning, then, leads to a paradoxical conclusion that the liar sentence is both true and false.

In this paper, I highlight some of the ways that logicians and philosophers have tried to solve the paradox. In particular, I explore the non-classical solutions to it 
couched in terms of a gappy logic and a glutty logic. According to the gappy solution, the liar sentence is neither true nor false, and the argument that leads to the paradox is logically unsound. On the other hand, according to the glutty solution, the liar sentence is indeed both true and false, but any subsequent reasoning that results from it is logically invalid. I show ways of motivating these views, and what they imply about the notions of truth and logical validity. Finally, I raise some relatively new problems that each view should address. Before that, I show how the liar paradox is generated by the standard view of truth and falsity, and explore several solutions to the paradox that preserve this standard view and the classical notion of validity.

\section{THE STANDARD VIEW OF TRUTH AND FALSITY}

While it is true that the sum of two odd numbers is an even number, it is simply false that the sum of two even numbers is an odd number. We all know this. But what exactly are we saying when we judge that some sentence is true or false? That is, in virtue of what is a sentence true or false?

One familiar story that logicians and philosophers have about truth and falsity is pretty straightforward: the sentence, 'The sum of two odd numbers is an even number' is true if and only if (iff) the sum of two odd numbers is an even number. On the other hand, the sentence, 'The sum of two even numbers is an odd number' is false iff the sum of two even numbers is not an odd number.

This standard view of truth is often cashed out in terms of the Tarskian truthschema:

$\mathrm{T}\langle\mathrm{P}\rangle \leftrightarrow \mathrm{P}$, (where $\mathrm{T}$ is the truth-predicate, ' $\leftrightarrow$ ' is the bi-conditional connective, $\mathrm{P}$ is replaced by any sentence and $\langle\mathrm{P}\rangle$ is a canonical name of that sentence (Eldridge-Smith 2018)).

On the other hand, the formal definition of falsity would be in terms of a falsityschema:

$\mathrm{F}\langle\mathrm{P}\rangle \leftrightarrow \sim \mathrm{P}$, (where $\mathrm{F}$ is the falsity-predicate and ' $\sim$ ' is the classical negation connective (Amour-Garb \& Beall 2005)).

Given these two notions, we could further define the falsity-predicate in terms of the truth-predicate via the equivalence:

$$
\mathrm{F}\langle\mathrm{P}\rangle \leftrightarrow \mathrm{T}\langle\sim \mathrm{P}\rangle .
$$

Given this, we could derive the following logical equivalences as well:

$$
\begin{aligned}
& \mathrm{T}\langle\mathrm{P}\rangle \leftrightarrow \sim \mathrm{T}\langle\sim \mathrm{P}\rangle ; \\
& \mathrm{F}\langle\mathrm{P}\rangle \leftrightarrow \sim \mathrm{F}\langle\sim \mathrm{P}\rangle ; \text { and }
\end{aligned}
$$




$$
\mathrm{T}\langle\mathrm{P}\rangle \leftrightarrow \mathrm{F}\langle\sim \mathrm{P}\rangle .
$$

The truth-schema entails the transparency principle, which tells us that 'sentences $\mathrm{P}$ and $\mathrm{T}\langle\mathrm{P}\rangle$ can be substituted for each other in any non-opaque context without changing the semantic value of a sentence of which they are part (or the whole)' Field (2008, 12).

We could take the transparency principle as entailment rules for introducing and eliminating the truth-predicate:

\section{[T-introduction] $\mathrm{P} \vdash \mathrm{T}\langle\mathrm{P}\rangle$;}

[T-elimination] $\mathrm{T}\langle\mathrm{P}\rangle \vdash \mathrm{P}$, (where ' $\vdash$ ' is the entailment symbol).

Thus, since the sentence, “'The product of two primes is a composite number' is true" is logically equivalent to the sentence, "The product of two primes is a composite number', we could always substitute one for the other in any non-opaque context. For example, in teaching the product of two primes to children, we could eliminate the truth-predicate and simply say, 'The product of two primes is a composite number' without the loss of cognitive meaning in the given context. By the same token, we could also introduce the truth-predicate in true sentences. For example, from the sentence 'The product of two primes is a composite number', we could also say that " "The product of two primes is a composite number' is true" or 'It is true that the product of two primes is a composite number.' Again, this inference is valid so long as the addition of the truth-predicate does not affect the sentence's cognitive meaning in the given context.

The same entailments hold for the falsity-schema as well.

$$
\begin{aligned}
& {[\text { F-introduction }] \sim \mathrm{P} \vdash \mathrm{F}\langle\mathrm{P}\rangle ;} \\
& {[\text { F-elimination }] \mathrm{F}\langle\mathrm{P}\rangle \vdash \sim \mathrm{P} .}
\end{aligned}
$$

Thus, since "The sum of two even numbers is an odd number" is false" is logically equivalent to 'The sum of two even numbers is not an odd number,' we could derive one from another in any non-opaque context without losing the semantic value of that sentence.

The transparency of truth and falsity involves three laws of logic: bivalence (LBV), excluded middle (LEM), and non-contradiction (LNC). (Technically, these are laws in classical logic (Shapiro and Kouri Kissel 2008). As we shall see later, however, to reject such 'laws' results to a kind of non-classical logic.) LBV tells us that there are two possible truth valuations for any sentence; they are either true or false. For example, given LBV, the sentence, 'The sum of an odd number and an even number is an odd number' and 'The sum of two even numbers is an odd number' are either true or false. As we all know, the former is true while the latter is false.

Related to LBV is LEM. It tells us that, for any sentence, either that sentence is true or its negation is true. Formally, for any sentence $P, T\langle P\rangle \vee F\langle P\rangle$ (where ' $V$ ' is the 
disjunction connective). These exhaust all truth possibilities; there cannot be a third way of evaluating the truth of a sentence. Thus, even if there is still no proof for the Goldbach conjecture, it is true that either every even integer greater than 2 can be expressed as a sum of two primes, or else not every even integer greater than 2 can be expressed as a sum of two primes. There cannot be another truth valuation for this.

Finally, LNC tells us that a sentence and its negation cannot both be true, since they are mutually exclusive. That is, for any sentence $P, F\langle T\langle P\rangle \wedge F\langle P\rangle\rangle$ (where ' $\wedge$ ' is the conjunction connective). Consider Goldbach's conjecture again. It cannot both be the case that every integer greater than 2 can be expressed as a sum of two primes and that not every integer greater than 2 can be expressed as a sum of two primes. If the former is true, then the latter must be false. On the other hand, if the latter is true, then the former must be false.

\section{THE LIAR PARADOX AND CLASSICAL SOLUTIONS TO IT}

The transparency principle and the laws of classical logic generate the liar paradox. There are many versions of the paradox throughout the history of philosophy. There is the version by Epimenides the Cretan concerning whether his declaration that 'All Cretans are liars' is true or not. There is also the version of Eubulides of Miletus asking whether the sentence, 'What I am saying now is a lie' is also true or not. ${ }^{1}$ But a more well-known version of the paradox starts with the liar sentence, 'This sentence is false'.

The argument that leads to the liar paradox is a proof by cases. Letting $\lambda$ be the liar sentence, it proceeds as follows:
(i) $\lambda \leftrightarrow F\langle\lambda\rangle$
(ii) $\mathrm{T}\langle\lambda\rangle \vee \mathrm{F}\langle\lambda\rangle$
premise
(iii) $T\langle\lambda\rangle$
LEM
(iv) $\lambda$
case one
(v) $F\langle\lambda\rangle$
(iii), T-elimination
(vi) $T\langle\lambda\rangle \wedge F\langle\lambda\rangle$
(i), (iv), bi-conditional-elimination
(vii) $\mathrm{F}\langle\lambda\rangle$
(iii), (v), conjunction-introduction
(viii) $\lambda$
case two
(ix) $\mathrm{T}\langle\lambda\rangle$
(i), (vii), bi-conditional-elimination
(viii), T-introduction
(x) $T\langle\lambda\rangle \wedge \mathrm{F}\langle\lambda\rangle \quad$ (ix), (vii), conjunction-introduction
(xi) $\mathrm{T}\langle\lambda\rangle \wedge \mathrm{F}\langle\lambda\rangle$
(ii), (vi), (x) disjunction-elimination ${ }^{2}$

What makes the liar paradox a problem for logicians and philosophers is the worry that from seemingly innocuous assumptions and a seemingly valid inference, we arrived at a contradictory conclusion that at least one sentence is both true and false. This is worrisome, since, given the classical logical law, ex contradictione quodlibet (ECQ), anything follows from a contradiction. This means that if we do accept that the liar sentence is both true and false, then we have to accept any trivial conclusion 
whatsoever. For example, given ECQ, the putatively false sentence, 'Every odd integer is the sum of two even integers' would logically follow from the liar sentence.

There are ways out of this conundrum that still preserve the laws of classical logic. ${ }^{3}$ One way is to reject the liar sentence and say that being self-referential, it is simply meaningless. One motivation for this idea is that truth and falsity could only be attributed to sentences with a complete thought, or to sentences that express a proposition. Since the liar sentence does not express a complete thought, it is simply meaningless. Given this, the liar paradox would not arise.

This way of responding to the liar paradox, however, is a nonstarter, since some self-referential sentences are meaningful. While it is true that the sentence, 'This is an English sentence' is self-referential, it still is a meaningful sentence. By the same token, the liar sentence is meaningful despite being self-referential. Self-referentiality, therefore, is not the issue at stake here.

Another way out of the liar paradox is the hierarchical response. This solution is famously defended by the logician, Alfred Tarski (Beall, Glanzberg \& Ripley 2017). According to the hierarchical solution, a given language forms a hierarchy of sentences. We have lower-level sentences which do not contain semantic predicates; we have higher-level sentences which do contain them. In this picture, the semantic predicates 'is true' and 'is false' are 'higher-order' predicates, which only applies to sentences at a lower level. For example, the sentence, 'The table is brown' is a sentence in a lower level, while "“This table is brown' is true" is a higher-level sentence that talks about the sentence at the lower level. This implies that the liar sentence is illformed because it violates the hierarchical ordering of sentences and misapplies the truth predicate.

One problem against the hierarchical response is that it leads to a counterintuitive result. Suppose $\mathrm{S}$ is a lower level sentence. Then ' $\mathrm{S}$ is true' would be a higher level sentence that talks about $S$. But we could iterate the process and have " $S$ is true' is true" at yet another higher-level sentence that talks about the lower level sentence, 'S is true', and so on. Since the semantic predicate can be iterated in this way, it leads to an infinite regress; thus making the notion of truth conceptually inert.

Furthermore, as the philosopher Saul Kripke (1975) has pointed out, it will be extremely hard to have various non-paradoxical sentences within the hierarchy. Suppose Alan says, 'Everything Brian says is true'. Alan's sentence must be located higher up the hierarchy than everything Brian says. But if Brian's sayings include the sentence, 'Everything Alan says is true', that sentence must appear higher in the hierarchy than everything Alan says. But this is simply impossible.

Finally, as Eldridge-Smith (2018) has recently pointed out, the hierarchical solution would not work for liar-like paradoxes that do not contain a semantic predicate. The Pinocchio paradox, first devised in 2001 by his then 11-year-old daughter, Veronique Eldridge-Smith, shows this. Consider the Pinocchio principle: Pinocchio's nose grows iff what Pinocchio is saying is false (or untrue). What happens if Pinocchio says, 'My nose is growing'? If his nose indeed grows, then what he says is true. But if so, then, given the Pinocchio principle, he must be saying something untrue. This cannot be the case, however, since his nose is in fact growing. But if he is saying something untrue, then his nose must not be growing. But it is. This now leads 
to a contradiction much like the liar paradox. Notice, however, that unlike the liar sentence, the sentence, 'My nose is growing' does not contain any semantic predicate. Yet, it still generates the liar-like paradoxes. ${ }^{4}$

\section{TRUTH GAPS AND REVENGE PARADOXES}

Perhaps, the right solution to the liar paradox involves rejecting the standard view of truth and falsity. This, in turn, implies rejecting at least one of the laws of classical logic. One such nonclassical solution is the gappy view. According to this view, the liar sentence is neither true nor false; it belongs to a class of sentences that are in the gap between truths and falsities. In admitting that there are sentences which are neither true nor false, gappy theorists reject both LBV and LEM, since they admit a third, neither true nor false, semantic value.

One motivation for the gappy view is the thought that while some sentences are determinately true and others are determinately false, there is a class of sentences which are indeterminately true and indeterminately false. For example, while the sentence, 'The Philippines is in Southeast Asia' is determinately true and the sentence, 'The Philippines is in Europe' is determinately false, some sentences are neither. Answers to presumptive questions and sentences about future contingents seem to fall under this gap category (Restall 2006, 62).

Consider the case of some answers to presumptive questions. If one were asked whether she has stopped beating her dog, a simple 'yes' or 'no' answer would seem inappropriate. If she answers, 'Yes, I have stopped beating my dog', then she has admitted that she has beaten her dog at least once in the past. On the other hand, if she answers, 'No, I have not stopped beating my dog', then she has admitted that she still beats her dog. But if she has never beaten her dog or she does not even have a dog, then neither sentence is determinately true nor determinately false. The sentence, 'I have stopped beating my dog' is neither true nor false.

Consider now the case of sentences about the future. A sentence about future contingents seems to be, at present, neither true nor false. Is the sentence, 'There will be an exam tomorrow' true at the moment of its utterance? The sentence is determinately true if there is an exam tomorrow; false if there is none. But at the time of its utterance, the sentence is neither true nor false, since it still awaits for a determinate truth value..$^{5}$

Given this, we could now sketch the gappy view's solution to the liar paradox. If the liar sentence is neither true nor false, then the argument that leads to the paradox is unsound. Thus, gappy theorists would now have a way to reject the conclusion of the liar paradox. Before this, let us first define what it is for an argument be logically valid and sound.

One way of defining the validity of arguments is via truth-preservation. To wit: an argument is valid iff for all possible cases, if the premises are true, then the conclusion is true. This notion is easy enough to understand. An argument is valid iff, for all possible ways of assigning a truth value to the premises and conclusion, there is no case where the argument's premises are true and its conclusion is false. Conversely, if there is a possible case where the argument has true premises but a false conclusion, 
then the argument is invalid. An argument is sound iff it is valid and has true premises. It is unsound if it is either invalid or has a false premise.

Since gappy theorists admit sentences which are neither true nor false, however, this simple view of validity becomes more complicated. According to one version of gappy logic, an argument is valid just in case, for all possible cases, if the premise has a designated value, then the conclusion has a designated value. ${ }^{6}$ A designated value is defined here as the value preserved in valid arguments. Since gappy theorists accept the truth-preservation idea of validity, it follows that, for them, only the semantic value, 'true' is designated. Thus, an argument is valid iff there is no case where the premises are designated, that is, has a semantic value, 'true', while the conclusion is not designated. Conversely, if there is such a case, then the argument is invalid. Furthermore, an argument is sound if it is valid and all of its premises are designated.

What of the liar paradox? On this version of the gappy view, the liar sentence is neither true nor false; it does not have a designated value. Since the liar sentence is not designated, the premises of the argument that leads to the paradoxical conclusion would not be designated as well. For example, line (ii) in the proof above would not be designated. Since the liar sentence is not designated, it follows that its negation will not be designated as well. Thus, making line (ii) neither true nor false. (This indeed follows since the gappy view rejects LEM.) This, then, makes the whole argument unsound.

The gappy view provides an interesting solution to the liar paradox in terms of truth gaps. But it is not without problems. One such problem is the so-called revenge paradoxes ((Beall 2008, ch. 1); (Garrett 2014, 157)). One version of a revenge paradox is the strengthened liar paradox. Suppose that instead of formulating the liar sentence in terms the falsity-predicate, we formulate it as: 'This sentence is not true.' Call this sentence $\lambda 1$. Since $\lambda 1$ does not use the falsity-predicate, we do not need the assumption of LBV or of LEM to generate the liar paradox. We only need a weaker assumption that $\lambda 1$ is true or is not true. The proof will be the same as before, except that all instances of $\lambda$ will be replaced by $\lambda 1$, and all instances of $\mathrm{F}\langle\lambda\rangle$ by $\mathrm{T}\langle\sim \lambda 1\rangle$. Informally, the argument proceeds as follows. If $\lambda 1$ is true, then $\lambda 1$ is not true; if $\lambda 1$ is not true, then $\lambda 1$ is true. From this, the paradoxical conclusion that $\lambda 1$ is both true and not true follows. The gappy view, therefore, still does not escape the paradox. ${ }^{7}$

\section{TRUTH GLUTS AND THE EXISTENCE OF DIALETHEIA}

Another non-classical solution to the liar paradox is the glutty view. According to this view, the liar sentence is both true and false; it belongs to a class of sentences, which can be called 'truth gluts'. While some sentences are true or false (or, perhaps, are in the gap), a few are gluts - they are both true and false. ${ }^{8}$ In admitting that some sentences are truth gluts, glutty theorists not only reject LBV, since sentences are either true, false, or are gluts; they also reject LNC, since some sentences can be both true and false. But could a particular sentence really be both true and false? The short answer is, Yes! Here we consider two such sentences: a sentence about motion and a sentence about making a choice. 
Consider the sentence, 'The car is moving'. If the sentence is true, then the car is moving from one location to another. That is, it is at a state of flux between the two location-points. But if motion implies this, then the moving car is and is not at one particular point. Thus, a sentence about motion implies that it is both true and false of a moving object that it is at some particular point. But if so, then the sentence about motion implies something that is both true and false.

Consider now the sentence, 'I am deciding whether to accept a certain hypothesis, H or not.' If this sentence is true, then I am at the moment of choice, where I am yet to accept $\mathrm{H}$. But if this is so, then it follows that I am in a state where I am yet to accept and, at the same time, yet to not accept $\mathrm{H}$. That is, I am in a contradictory state of accepting and not accepting H. Again, if this is so, then a sentence about making a choice implies something that is both true and false.

Granted that some sentences are truth gluts, but how does this fact provide a solution to the liar paradox? Again, for glutty theorists, the liar sentence is both true and false. As such, they would wholly accept the conclusion of the liar paradox. Be that as it may, however, they would still disallow any trivial inference from the liar sentence, since, in this glutty picture, ECQ is an invalid inference. We take these two ideas in turn.

Like gappy theorists, glutty theorists define logical validity in terms of the preservation of a designated value from premise to conclusion. They differ, however, because, for glutty theorists, the semantic value, 'both true and false' will be designated as well. Thus, for glutty theorists, an argument is valid iff, for all possible cases, if the premise is either true or both true and false, then the conclusion is either true or both true and false as well. That is, there is no case where the premises are designated and the conclusion is not.

How does this solve the liar paradox? Given their theory of validity, glutty theorists would simply accept the contradictory conclusion that follows from the liar argument. In this way, the paradox would be wholly accepted as well. This is right, since glutty theorists, like Priest, accepts a kind of paraconsistent logic known as the logic of paradox ((Priest 2008, ch. 7.4); (Sider 2010, ch. 3.4.4)).

One might argue, however, that since the liar sentence is both true and false, the argument that results from it must be unsound. It is unsound, since, though valid, the liar argument has a false premise. This false premise is none other than the liar sentence that says of itself that is both true and false.

Glutty theorists might reply that since the semantic value, 'both true and false' is preserved in valid arguments, we would need to revise the notion of soundness as well. They could define soundness as follows. If the argument is valid in the glutty sense, then if all of its premises are either actually true or actually both true and false premise, then the argument is sound as well. Given this revised notion of soundness, glutty theorists would say that the liar argument is both valid and sound.

If glutty theorists accept that the liar sentence is both true and false, however, then what of trivial conclusions that might be inferred from it via the ECQ inference? That is, if the liar sentence is both true and false, then, via ECQ, any putatively false conclusion, like $1=0$, would follow. 
Glutty theorists might respond that given their theory of validity, ECQ is actually invalid. Formally, ECQ tells us that $\mathrm{P} \wedge \sim \mathrm{P} \vdash \mathrm{Q}$. That is, from a contradictory sentence, ' $\mathrm{P} \wedge \sim \mathrm{P}$ ', we could derive any trivial conclusion, ' $\mathrm{Q}$.' This is a classically valid inference in that there is no possible case where its premise is true and its conclusion is false. Since the glutty theorists allow truth gluts, however, there will be a case where ECQ fails - a case where $P$ is both true and false (a truth glut) and Q is false. That is, there is a possible case where the contradictory sentence, ' $P \wedge \sim P$ ' is designated, but the conclusion, ' $Q$ ' is not. Hence, according to this glutty logic, ECQ would be invalid.

Despite the deliverances of and the motivations for the glutty view, however, one could still raise worries against it. One worry is about the plausibility of there being dialetheia. That is, whether there are sentences which are really both true and false. It is simply difficult to make conceptual sense of how sentences could be unqualifiedly both true and false (Garrett 2014, 157).

Let us suppose, however, that we could make sense of the existence dialetheia, the next question is whether it is rational to believe there are such things. Priest has argued that 'If a rational agent has good evidence for something, then she ought to accept it' (Priest 2006, 91) and since we do have good evidence that there are sentences which are both true and false, it is rational to believe that there are.

Having good evidence for a belief, however, is not the only constraint for the rationality of beliefs; there is also the so-called doxastic regularity principle. The principle tells us that 'If something is doxastically possible for a rational agent, then that agent has to assign a positive credence to it' (Hájek ms 2012; Easwaran 2014). Doxastic possibility is characterized as any sentence that is a live possibility given what a rational agent believes, and subjective probability as a measure of an agent's credence or degree of belief towards that sentence. Thus, if the belief that it will rain today is a live possibility for an agent, then, given regularity, the agent's credence to it must be positive. If, on the other hand, the agent's credence to that belief is 0 , then it is not a live possibility for that agent.

Given the regularity constraint on rational beliefs, a dialetheist (glutty theorist) would be confronted with a complex dilemma. Either her belief is not doxastically possible even for her or else she must assign positive credence to the sentence that true contradictions exist. The former makes her belief self-defeating. The latter seems to compel her to choose between a number of more fine-grained options, each of which seem undesirable for a regular and rational theory of probability. ${ }^{9}$

\section{CONCLUSION}

The liar paradox remains to be one of the most enduring puzzles in the canons of philosophy. It has continually challenged our concept of truth and our notion of logical validity. As discussed, standard solutions to the paradox that aim to preserve the classical notion of truth and validity succumbed to further problems and puzzles. Despite their deliverances, the gappy and the glutty solutions that were considered 
have likewise invited more philosophical questions. All of this, however, must not be taken in an entirely negative light. As we have seen, almost all the technical developments in logic have coincided with a solution to the paradox. Tarski's Tschema, Łukasiewicz's three-valued (gappy) logic, and Priest's (glutty) logic of paradox have resulted from a formal solution to the paradox Arguably, the more perplexing the paradox becomes, the more sophisticated the logical machinery that develops. Perhaps, this is the true legacy of the liar paradox.

\section{NOTES}

1. For a discussion of the different versions of the liar paradox, see (Sorensen 2003, ch. 7) and (Beall, Glanzberg, \& Ripley 2019).

2. Compare this formulation of the liar paradox with (Beall 2008), (Cobreros, et al. 2013), and (Eldridge-Smith 2018).

3. These solutions are standard as well. Compare the discussion here with (Garrett 2014, 156-160).

4. The Pinocchio paradox has blossomed into a growing literature since the publication of (Eldridge-Smith \& Eldridge-Smith 2010). For a detailed summary of the responses and replies to them, see (Eldridge-Smith 2018).

5. If $\mathrm{B}$ theorists or eternalists are right that the future is as real as the present and the past, then, arguably, sentences about future contingents have a determinate truth value; they are either true or false. The question about the reality of the future, however, is contentious. For a discussion of this issue, see (Garret 2011, 115).

6. There are different versions of a gappy logic. The version adopted here is $Ł 3$, named after its creator the Polish logician Jan Łukasiewicz. Compare the discussions here with (Priest 2008, sec. 7.3), (Restall 2006, 59-60), and (Sider 2010, sec. 3.4.1).

7. Of course, friends of the gappy view have responses to the revenge paradox; see (Beall 2008) for a discussion of some these responses.

8. The glutty view is also known as dialetheism. Priest is one of the main defenders of truth gluts, and he offers several arguments for the view; for a discussion, see (Priest 2006, ch. 3).

9. The rationality of the dialetheic commitment is an open topic worth pursuing in a future study.

\section{REFERENCES}

Amour-Garb, Bradley \& Jc Beall, eds. 2005. Deflationary Truth. Chicago: Open Court Publishing Company.

Beall, Jc, Michael Glanzberg, \& David Ripley. 2019. Liar Paradox. The Stanford Encyclopedia of Philosophy. E. N. Zalta (ed.), URL = <https://plato.stanford.edu/archives/fall2017/entries/liar-paradox/>. Accessed 19 September 2018.

Beall, Jc, ed. 2008. Revenge of the Liar. Oxford: Oxford University Press. 
Cobreros, Pablo, Paul Egré, David Ripley, \& Robert van Rooij. 2013. Reaching Transparent Truth. Mind. 122: 841-866.

Easwaran, Kenny. 2014. Regularity and Hyperreal Credences. Philosophical Review 123: $1-41$.

Eldridge-Smith, Peter. 2018. Pinocchio against the Semantic Hierarchies. Philosophia 48: 817-830.

Eldridge-Smith, Peter \& Veronique Eldridge-Smith. 2010. The Pinocchio Paradox. Analysis 70: 212-215.

Field, Hartry. 2008. Saving Truth from Paradox. Oxford: Oxford University Press.

Garrett, Brian. 2014. Elementary Logic. London: Routledge.

Garrett, Brian. 2011. What is this thing called Metaphysics?, 2nd edition. London: Routledge.

Hájek, Alan. ms. Staying Regular?. 2012 version available at URL = <http://fitelson.org/few/hajek_paper.pdf.> Accessed 19 September 2018.

Kripke, Saul. 1975. Outline of a Theory of Truth. The Journal of Philosophy 72: 690716.

Restall, Greg. 2006. Logic: An Introduction. London: Routledge.

Priest, Graham. 2008. An Introduction to Nonclassical Logic, 2nd edition. Cambridge: Cambridge University Press.

Priest, Graham. 2006. Doubt Truth to be a Liar. Oxford: Oxford University Press.

Priest, Graham. 1979. Logic of Paradox. Journal of Philosophical Logic 8: 219-241.

Shapiro, Stewart \& Teresa Kouri Kissel. 2008. Classical Logic. The Stanford Encyclopedia of Philosophy (Spring 2018 Edition), E. N. Zalta (ed.), URL = $<$ https://plato.stanford.edu/archives/spr2018/entries/logic-classical/>. Accessed 19 September 2018.

Sider, Theodore. 2010. Logic for Philosophy. Oxford: Oxford University Press.

Sorensen, Roy. 2003. A Brief History of the Paradox: Philosophy and the Labyrinths of the Mind. Oxford: Oxford University Press.

\section{ACKNOWLEDGEMENTS}

A version of this paper was presented at the DLSU PILOSOPO Big Lecture Series held in De La Salle University, Manila, Philippines on October 29, 2018. My thanks go to the organizers and participants of that event. I owe a special debt of gratitude to Hazel T. Biana, Ben Blumson, Peter Eldridge-Smith, Brian Garrett, Napoleon Mabaquiao, Jr., and the referees of this journal for the comments and suggestions that greatly improved this paper.

Submitted: 17 September 2019; revised 30 April 2020 